\title{
Actualizing High-Energy Bipolar-Stacked Solid-State Battery with Low-Cost Mechanically Robust Nylon Mesh- Reinforced Composite Polymer Electrolyte Membranes
}

Chao $X u^{1}$, Yujie Jiang ${ }^{1}$, Kang $X u^{1}$, Zhihong Chen ${ }^{2}$, Wanying Chang ${ }^{1}$, Nannan Cen

1, Jiaxi $\mathrm{Ni}^{1}$, Rui Xu ${ }^{1}$, Yuedong Huang ${ }^{2}$, Patimai Abulaiti ${ }^{2}$, Ayizulamu Tuohetimait

2, Chunyan Lai 1, *, Chengxin Peng 2,3, *

${ }^{1}$ C. Xu, Y. Jiang, K. Xu, W. Chang, N. Cen, J. Ni, R. Xu, C. Lai ${ }^{*}$

Shanghai Key Laboratory of Materials Protection and Advanced Materials in Electric Power

Shanghai University of Electric Power

Shanghai 200090, China

E-mail:laichunyan@shiep.edu.cn

${ }^{2}$ Z. Chen, Y. Huang, P. Abulaiti, A. Tuohetimait, C. Peng*

School of Materials Science and Engineering

University of Shanghai for Science and Technology

Shanghai 200093, China

E-mail: cxpeng@usst.edu.cn

${ }^{3}$ Key Laboratory of Advanced Energy Materials Chemistry (Ministry of Education)

College of Chemistry

Nankai University

Tianjin 300071, China 
Cathode

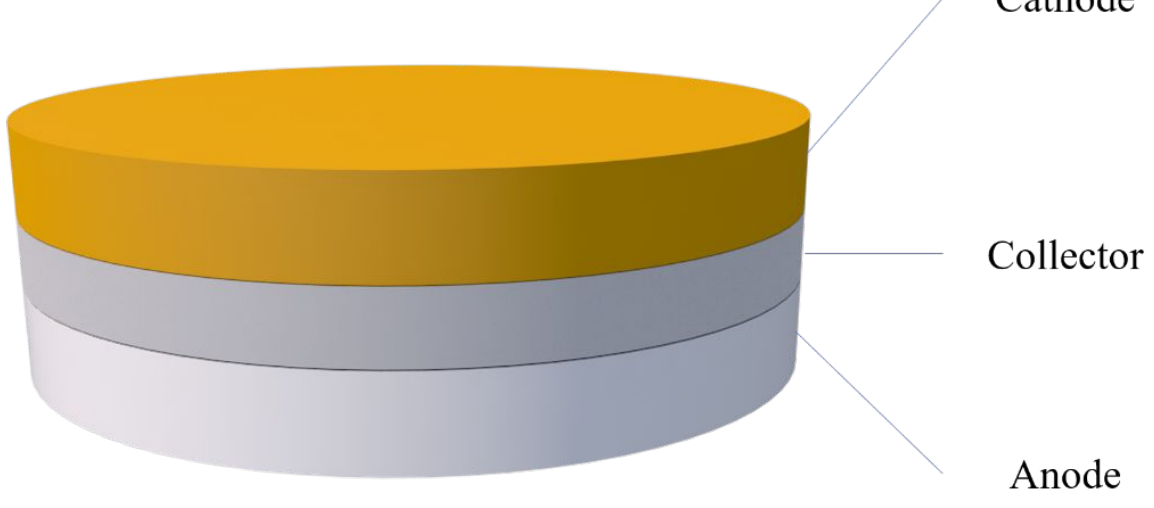

Figure S1. Schematic diagram of the configuration of the bipolar electrode. 


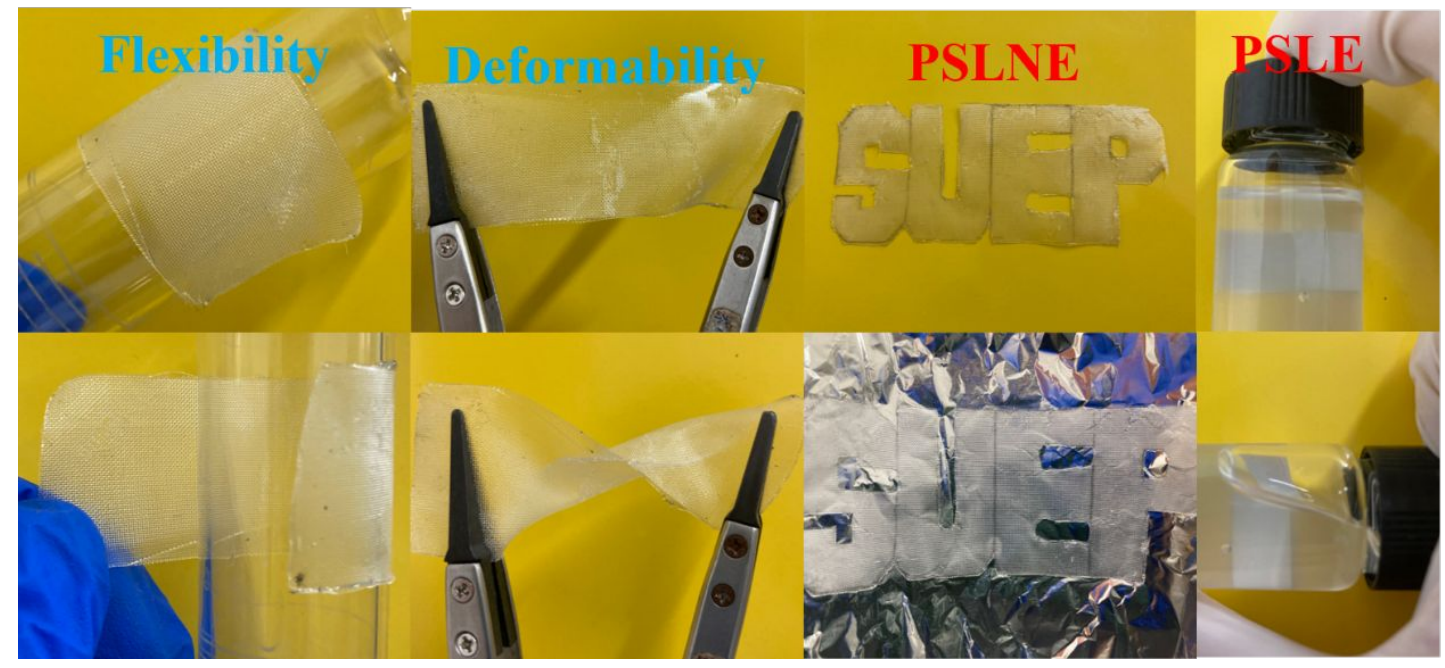

Figure S2. Schematic diagram of flexibility and deformability of PSLNE. 

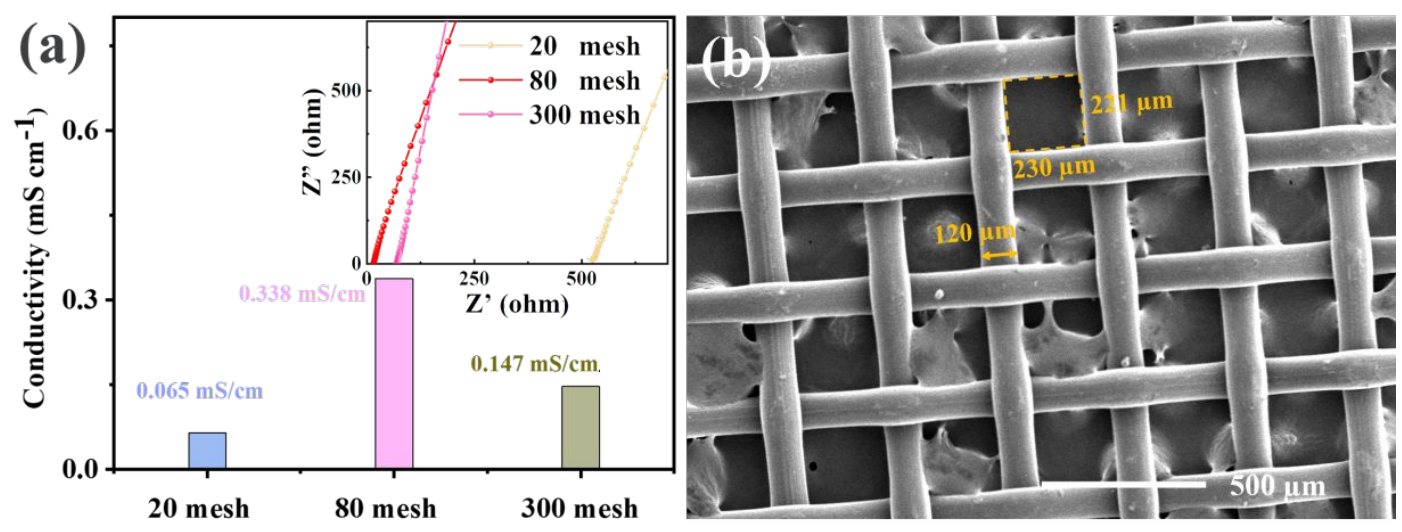

Figure S3. (a) The ionic conductivity of 20-, 80-, and 300-mesh nylon mesh compounded gel electrolyte at $30{ }^{\circ} \mathrm{C}$. Inset: the EIS curves. (b) SEM image of support net (80-mesh nylon mesh). 


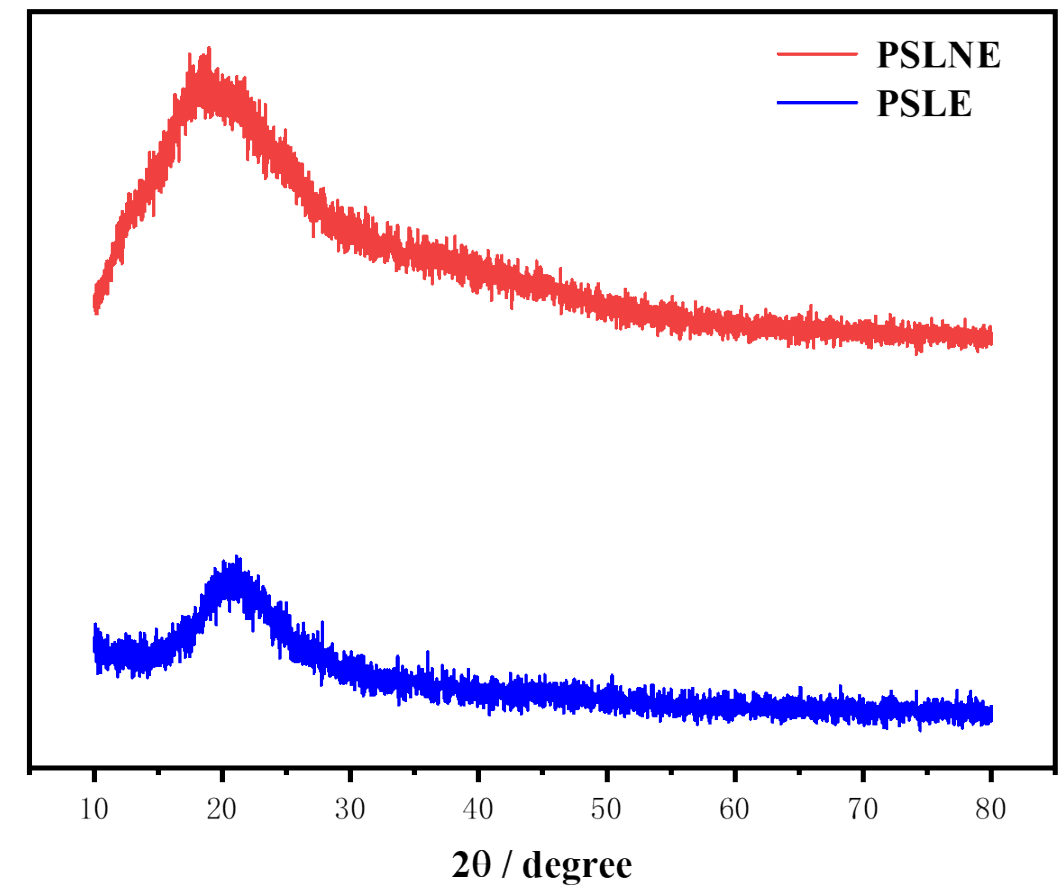

Figure S4. XRD patterns of the PSLNE and PSLE. 


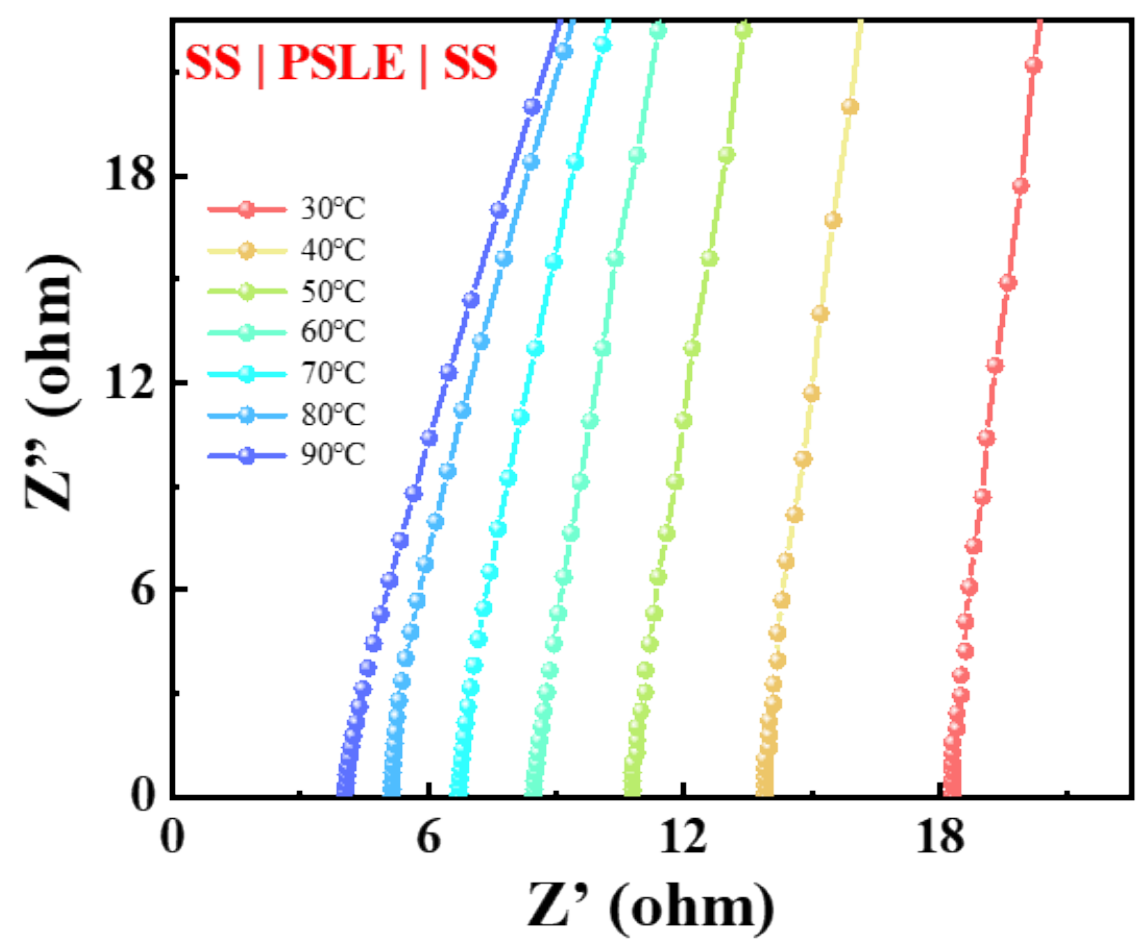

Figure S5. EIS curve of PSLE from $30-90^{\circ} \mathrm{C}$. 


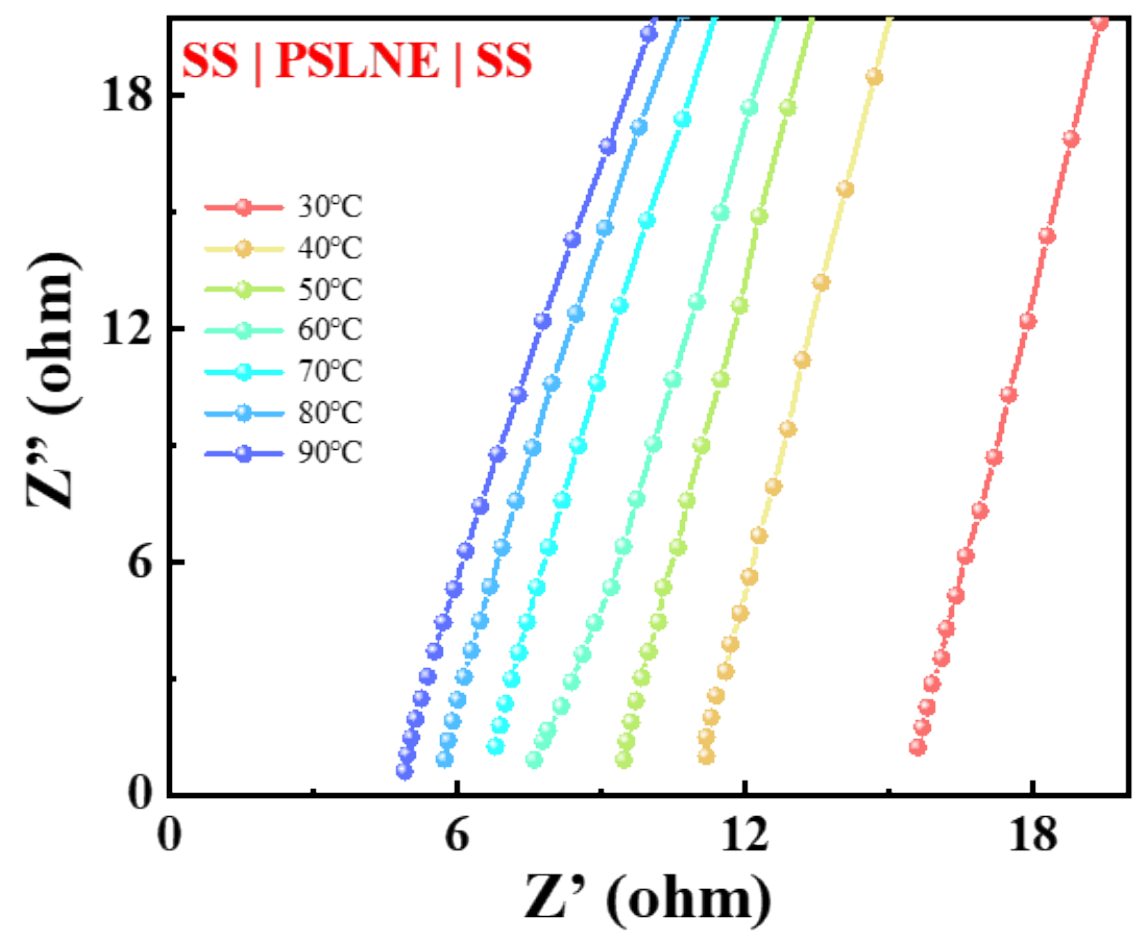

Figure S6. EIS curves of PSLNE from $30-90{ }^{\circ} \mathrm{C}$. 


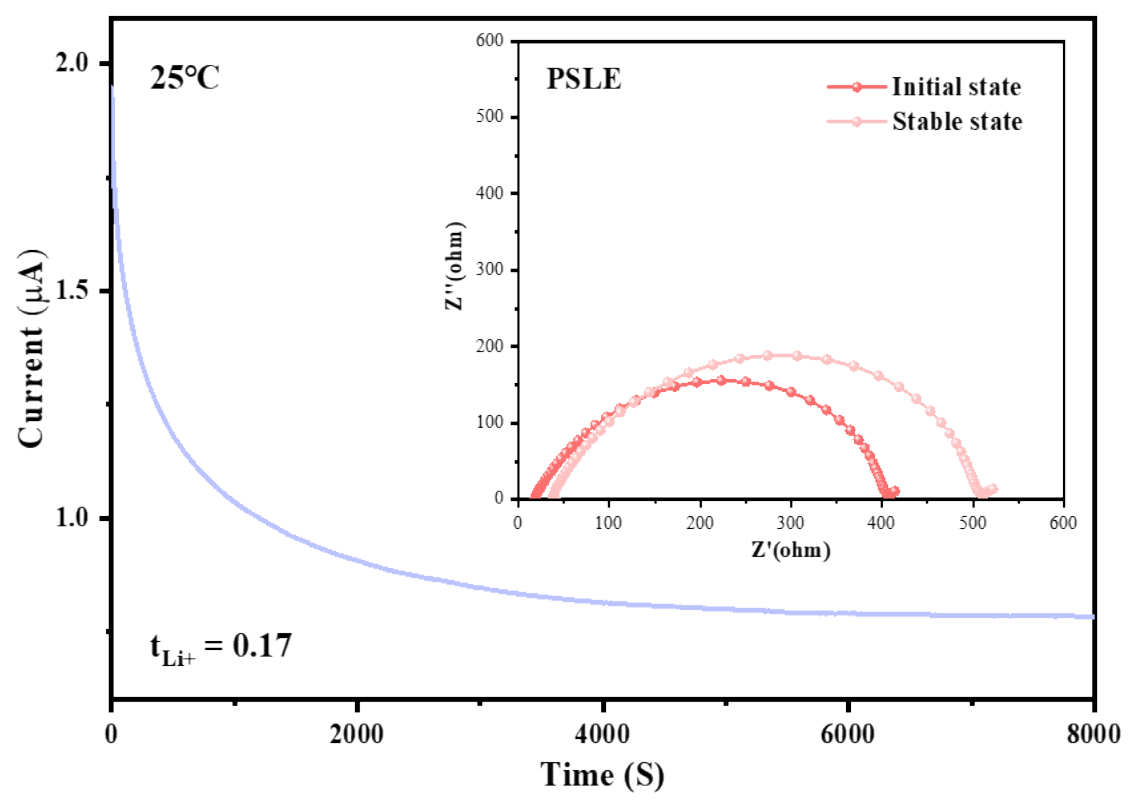

Figure S7. Chronoamperometry curve of the Li/PSLE/Li cell at a potential step of 10 $\mathrm{mV} \mathrm{s}^{-1}$ under $25^{\circ} \mathrm{C}$. Inset: the EIS curves before and after polarization of PSLE. 


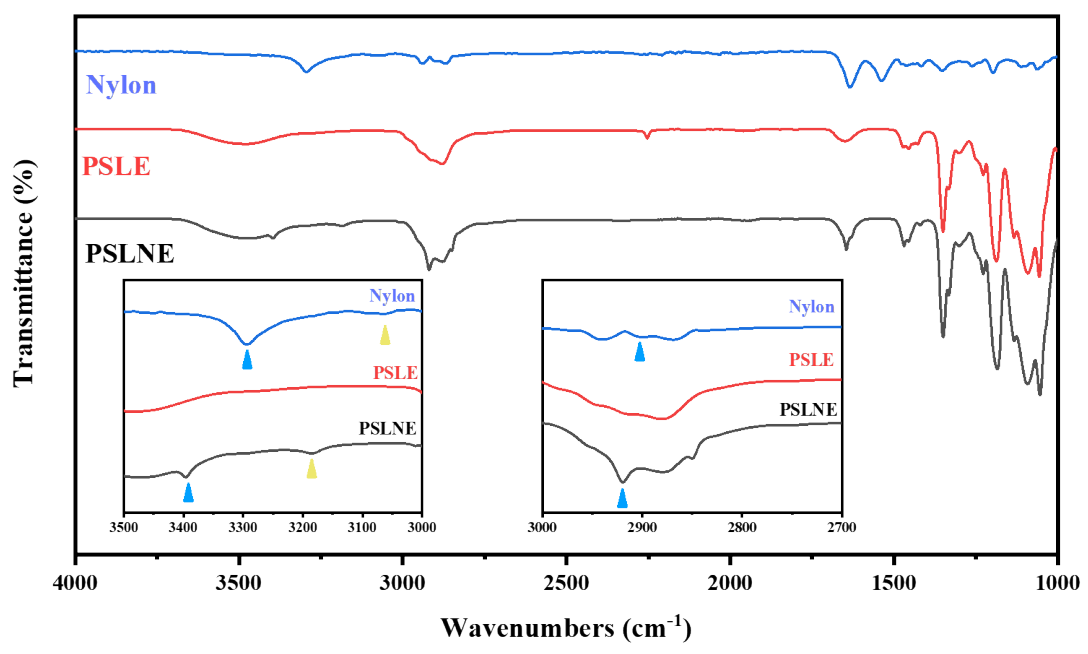

Figure S8. FTIR spectra of nylon mesh, PSLE and PSLNE. 


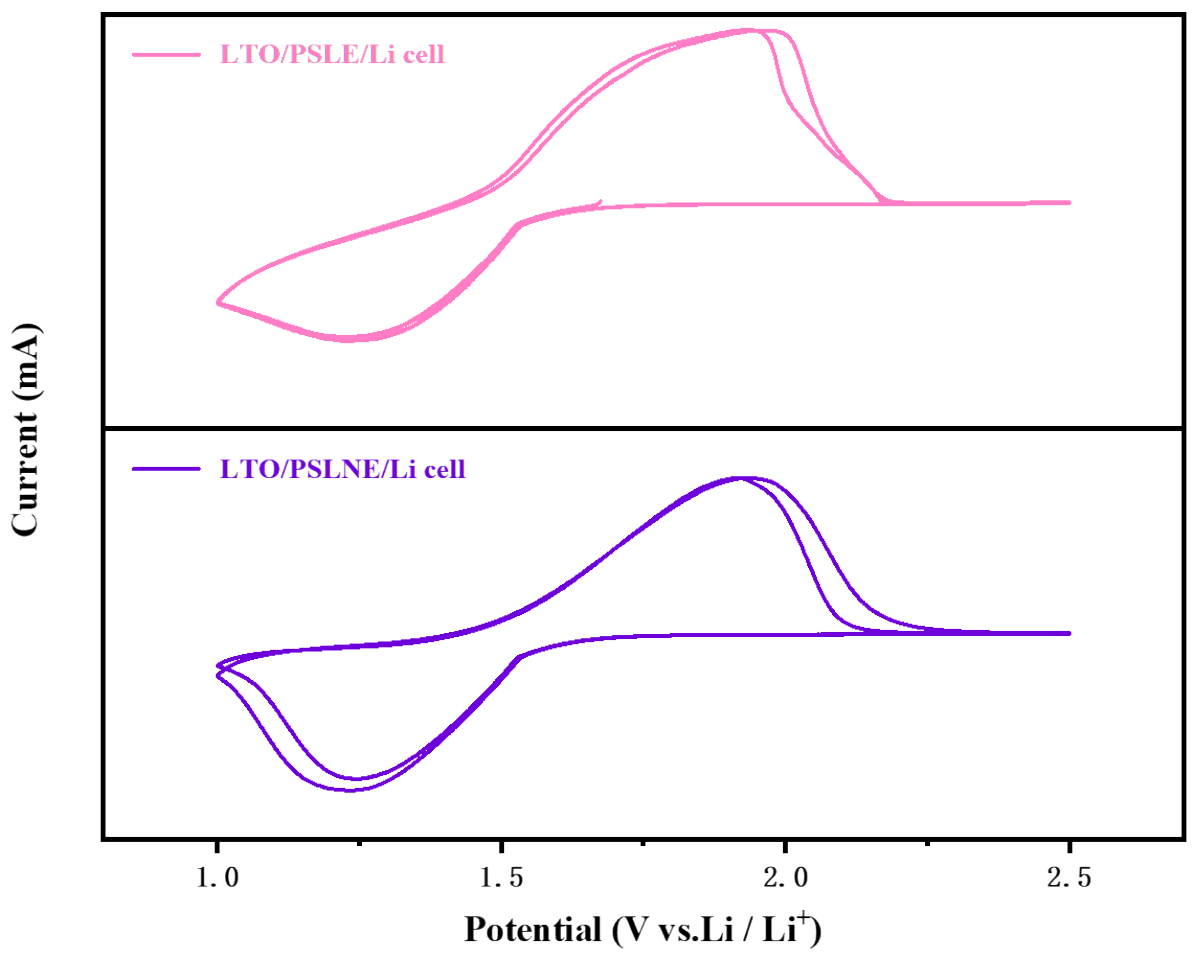

Figure S9. Cyclic voltammetric curves of LTO//Li cell. 


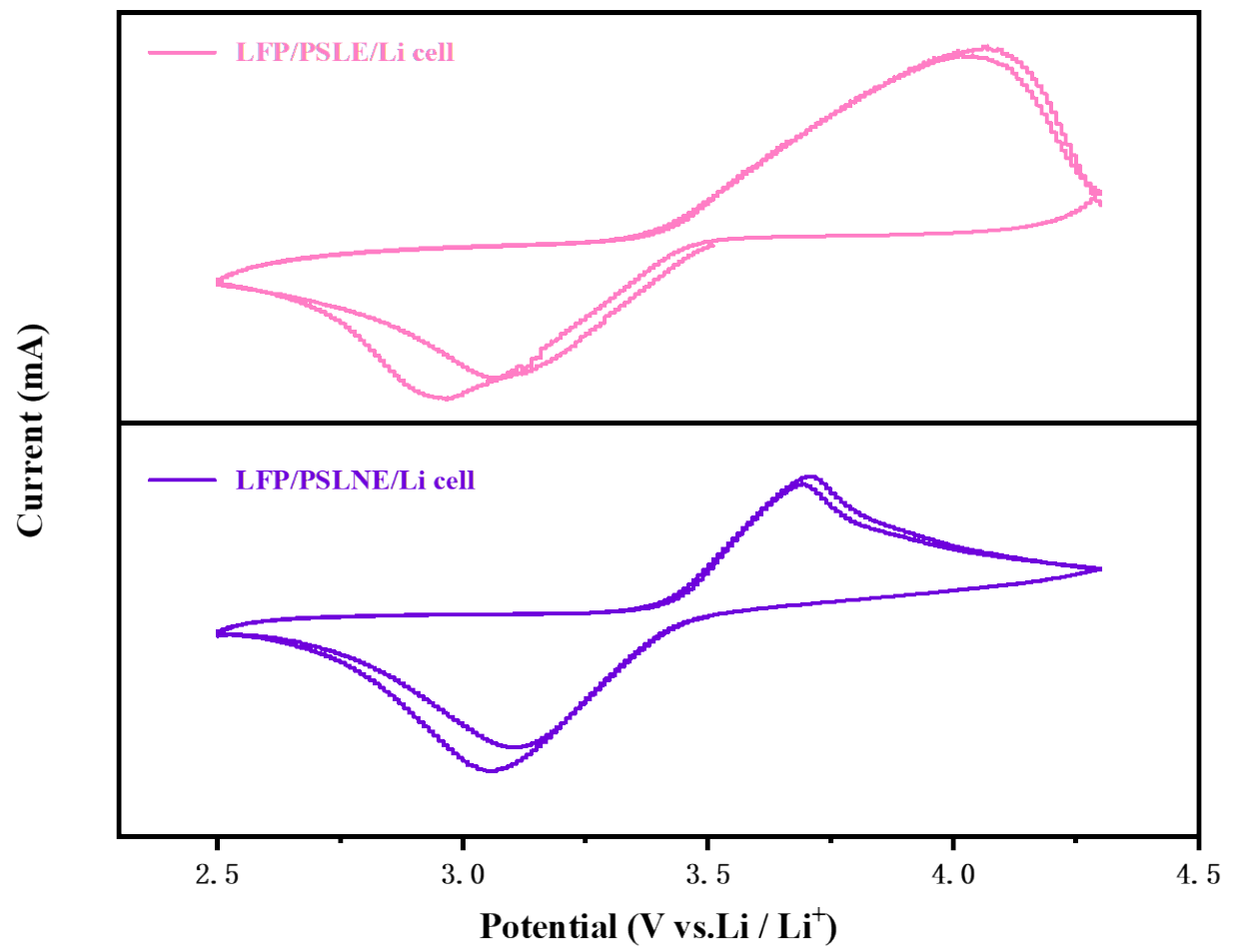

Figure S10. Cyclic voltammetric curves of LFP//Li cell. 

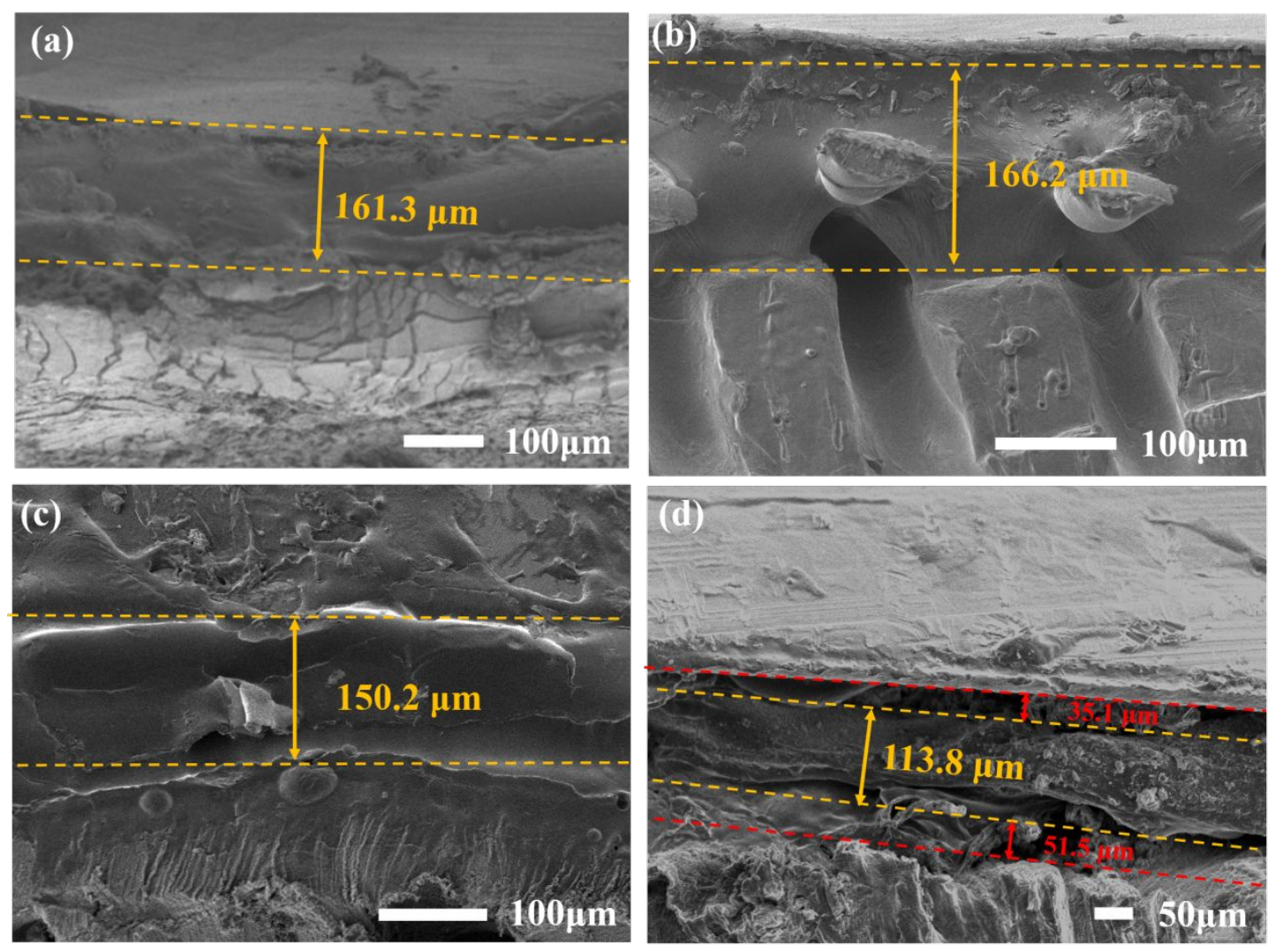

Figure S11. a) \& b) The cross-sections of the LTO/PSLNE/Li before and after cycles. c) \& d) Cross-sectional of LTO/PSLE/Li before and after cycles. 


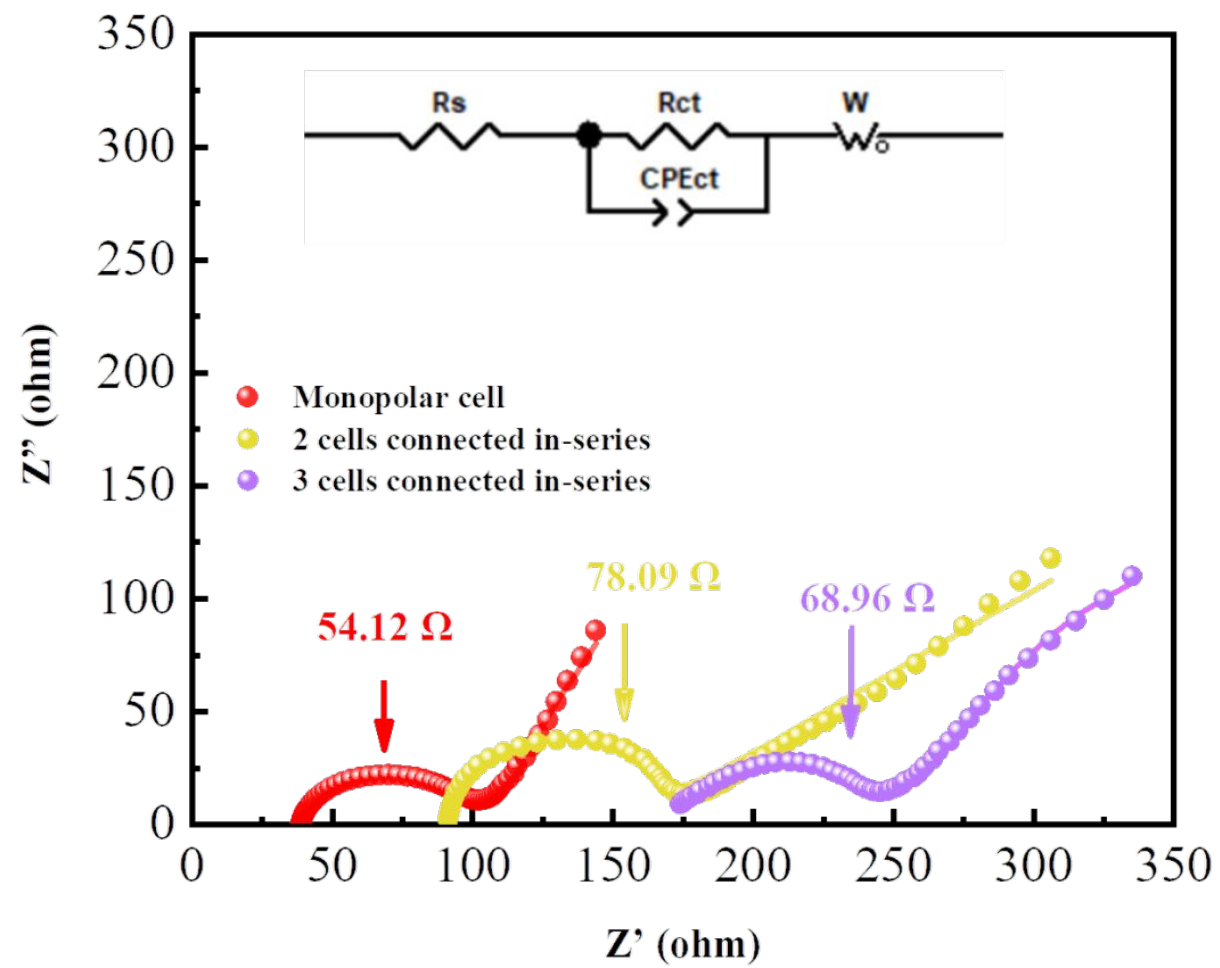

Figure S12. EIS curve of the monopolar cell and bipolar stacked cells ( 2 cells connected in-series and 3cells connected in-series). 